\title{
The stochastic finite element method in the assessment of bridge infrastructure objects - review
}

\author{
Piotr Owerko, ${ }^{1, *}$ \\ ${ }^{1}$ Faculty of Materials, Civil and Environmental Engineering, University of Bielsko-Biala, 43-309, st. \\ Willowa 2, Poland
}

\begin{abstract}
The stochastic finite element method (SFEM) is an extension of classical FEM which allows the representation of various types and sources of uncertainty in one computational system. This review paper presents information regarding SFEM implementation in the assessment of bridge objects. A concise theoretical background of the three most commonly used branches of SFEM is also presented. This technique is used in the assessment of bridge structures with regards to, e.g. load in motion problems, wind and seismic excitation analysis, random material property analysis, reliability and fatigue reliability analysis. However, it seems that the main feasibility concern is the lack of proper SFEM implementation into general purpose FEM systems which are used by bridge engineers. This is why, arguably, one of the most effective ways to introduce SFEM in real-life bridge engineering problems is with the methods that rely on multiple calculations of classical deterministic FEM e.g. Monte Carlo Simulation, or Response Function Method. To introduce randomness directly into individual finite elements of the model in a complete SFEM procedure it is usually necessary to develop proprietary computer programs.
\end{abstract}

\section{Introduction}

Bridge objects are key components of road infrastructure systems. Any serviceability failures of these structures often result in extremely costly renovation programs and troublesome hindrances to traffic flow. This consequences can seriously affect the transportation system efficiency of major cites especially when a failure concerns bridges in the city bypass or other fundamental communication routes.

To minimize the probability of the occurrence of these problems, special attention should be paid to the design stage of new bridges and thorough the assessment of existing ones prior to, e.g. renovation to increase their load capacity. Design costs are a very small fraction of the overall structures life cycle costs [1]. Thus, it is worthwhile investing time and measures for deep and reliable design or assessment analyses. For that purpose, in recent decades, probabilistic tools are more and more confidently implemented. Thanks to this, it is possible to properly represent important uncertainties in the analysis such us: random material

\footnotetext{
*Corresponding author: powerko@ath.bielsko.pl, piotrowerko@gmail.com
} 
properties, random loads and dynamic excitations, random executive imperfections and others.

An important branch of these methods is the Stochastic Finite Element Method (SFEM) [2,3] which is an extension of its deterministic ancestor FEM. Depending on the technique of uncertainty propagation representation, it is developed in several variants, mainly: (I) FEM with Stochastic Perturbation Method (hereinafter referred to as Perturbation SFEM) [4], (II) Spectral Stochastic Finite Element Method (SSFEM) [5], (III) FEM with Monte Carlo Simulation (MCS) Technique often with a supplement of Latin Hypercube Sampling (LHS) or other sampling techniques [6]. These methods have recently been implemented in the analysis of various types of bridge superstructures and in the aspect of different types of analysis (modal, fatigue, static, reliability, etc.)

Therefore, the main objective of this paper is to collect and analyse information regarding: (I) a concise outline of theoretical background of individual SFEM techniques, (II) a review of analyses and their types in which SFEM was effectively implemented for the assessment of bridge structures response, (III) the identification of the aspects in which SFEM requires further research and development or is currently infeasible in real-life engineering problems.

\section{Theoretical background}

\subsection{Random finite element method with perturbation approach}

In the perturbation method, Taylor series expansion is used to formulate relationships between chosen characteristics of a random response and random structural parameters. Instability of the approximate solution appears in higher order terms [5]. One of the first applications of the perturbation approach for engineering problems can be found, for example, in [7]. In [8] and [9] this approach was further developed to be compatible with FEM. The second-order version of the stochastic finite element method was practically implemented in the aspect of structural analysis by Kleiber and Hien [4].

Using this approach to, for example, introduce random material properties into the system will result in the following form of the stiffness matrix [2]:

$$
K=K_{0}+\sum_{i=1}^{N} K_{i}^{I} \propto_{i}+\frac{1}{2} \sum_{i=1}^{N} \sum_{j=1}^{N} K_{i j}^{I I} \propto_{i} \propto_{j}+\cdots
$$

where: : $\alpha_{i}(i=1,2, \ldots, N)$ are zero mean random variables representing system uncertainties after random field discretisation; and $K_{0}$ is a matrix containing expected values. $K_{i}^{1}$ and $K_{i j}^{I I}$ can be derived as follows:

$$
\begin{gathered}
K_{i}^{I}=\left.\frac{\partial K}{\partial \propto_{i}}\right|_{\propto=0} \\
K_{i j}^{I I}=\left.\frac{\partial^{2} K}{\partial \propto_{i} \partial \propto_{j}}\right|_{\propto=0}
\end{gathered}
$$

Analogical considerations can be made in the case of the uncertainty in loads:

$$
P=P_{0}+\sum_{i=1}^{N} P_{i}^{I} \propto_{i}+\frac{1}{2} \sum_{i=1}^{N} \sum_{j=1}^{N} P_{i j}^{I I} \propto_{i} \propto_{j}+\cdots
$$

and displacements:

$$
U=U_{0}+\sum_{i=1}^{N} U_{i}^{I} \propto_{i}+\frac{1}{2} \sum_{i=1}^{N} \sum_{j=1}^{N} U_{i j}^{I I} \propto_{i} \propto_{j}+\cdots
$$


The displacement vector $U$ can also be calculated using an iterative scheme [3]:

$$
\begin{gathered}
U_{0}=K_{0}^{-1} P_{0}, \\
U_{i}^{I}=K_{0}^{-1}\left(P_{i}^{I}-K_{i}^{I} U_{0}\right) \\
U_{i j}^{I I}=K_{0}^{-1}\left(P_{i j}^{I I}-K_{i}^{I} U_{i}^{I}-K_{j}^{I} U_{j}^{I}-K_{i j}^{I I} U_{0}\right),
\end{gathered}
$$

where: $U_{0}$ is equal to deterministic nodal displacements, $U_{i}^{I}, U_{i j}^{I I}$ are the first and secondorder perturbation of the displacement vector, respectively.

In the case of the assumption that random variables $\alpha_{i}, \alpha_{j}$ are Gaussian, the approximation of the response variability can be adopted as follows:

$$
\begin{gathered}
E(U) \approx U_{0}+\frac{1}{2} \sum_{i=1}^{N} \sum_{j=1}^{N} U_{i j}^{I I} \operatorname{Cov}\left[\propto_{i}, \propto_{j}\right] \\
\operatorname{Cov}[U, U] \approx \sum_{i=1}^{N} \sum_{j=1}^{N} U_{i}^{I}\left(U_{j}^{I}\right)^{T} \operatorname{Cov}\left[\propto_{i}, \propto_{j}\right]
\end{gathered}
$$

where: $E(U)$ and $\operatorname{Cov}[U, U]$ are the approximations of the expected values and covariance matrices of the random response (displacement), respectively.

The main drawback of second-order calculations is that it can only reliably represent phenomena with relatively low variability (coefficient of variation $<15 \%$ ). In other cases higher order approximations must be used. This led to development of the recursive stochastic finite element method [10] and the generalized perturbation method with the response function method which are presented in [11]. The latter was done with the use of the advanced symbolic Maple ${ }^{\circledR}$ system, and was supported by several numerical examples.

\subsection{Spectral Stochastic Finite Element Method (SSFEM)}

The Spectral Stochastic Finite Element Method (SSFEM) was introduced by Ghanem and Spanos [5]. SSFEM is a development of the deterministic FEM to accommodate random functions. One of the main goals of this method is to represent random material properties in a more computationally efficient manner than Monte-Carlo simulation. In [5] a random, special variation of material properties in a structure as described by a Gaussian stochastic field which is generated using Karhunen-Loève (K-L) expansion (presented in e.g. [3], initially derived in $[12,13,14])$ :

$$
f(x, \theta)=\bar{f}(x)+\sum_{i=1}^{\infty}\left[\xi_{i}(\theta) \sqrt{\lambda_{i}} \psi_{i}(x)\right]
$$

where: $\bar{f}(x)$ is the mean of random field/process, $\xi_{i}(\theta)$ is a group of uncorrelated random variables, $\lambda_{i}$ and $\psi_{i}(x)$ are eigenvalues and eigenfunctions of the covariance kernel, respectively.

On this basis, local (element) stiffness matrices in SSFEM are in the following form [3]:

$$
K^{e}(\theta)=K_{0}^{e}+\sum_{i=1}^{\infty} K_{i}^{e} \xi_{i}(\theta)
$$

where: $K_{0}^{e}$ is mean value of $K^{e}(\theta)$ and $K_{i}^{e}$ is given by:

$$
K_{i}^{e}=\sqrt{\lambda_{i}} \int_{\Omega_{e}} \psi_{i}(x) B^{T} D_{0} B d \Omega_{e}
$$

where: $D_{0}$ is the mean value of the constitutive matrix, while $B$ is the strain-displacement matrix. 
After aggregation of local stiffness matrices into a global form (using rectangular permutation matrices known from classical FEM), and with the assumption that loading is deterministic, the equilibrium equation is as follows:

$$
\left[K_{0}+\sum_{i=1}^{\infty} K_{i} \xi_{i}(\theta)\right] U(\theta)=F
$$

Alas, the covariance function of solution $U(\theta)$ (which is the random vector of nodal displacements) and corresponding eigenfunctions are unknown. Therefore, unlike in perturbation FEM, SSFEM requires using a second type of extension to express the response process (e.g. displacement). In [5] improved Neumann Expansion or/and Homogenous Chaos Expansions are used and compared. In the case of the latter one, the appropriate expression is as follows:

$$
\mathrm{U}(\theta)=a_{0} \Gamma_{0}+\sum_{i 1=1}^{\infty} a_{i 1} \Gamma_{1}\left(\xi_{i 1}(\theta)\right)+\sum_{i 1=1}^{\infty} \sum_{i 2=1}^{i 1} a_{i 1 i 2} \Gamma_{2}\left(\xi_{i 1}(\theta), \xi_{i 2}(\theta)\right)+\ldots
$$

where: $\Gamma_{n}\left(\xi_{i 1}(\theta), \ldots, \xi_{i n}(\theta)\right)$ is the $n$-th order polynomial chaos of the variables $\left(\xi_{i 1}(\theta), \ldots, \xi_{\text {in }}(\theta)\right)$. The above is often re-written into an alternative form by limiting series to $p$ terms and mapping functional $\Gamma_{n}($.$) into \Psi_{j}($.$) and coefficients a_{i}$ into $U_{j}$ in one-to-one manner:

$$
U(\theta)=\sum_{j=0}^{p} U_{j} \Psi_{j}(\theta)
$$

On the basis of eq. 10 to eq. 14, the final equilibrium equation is constructed and solved as a linear system of algebraic equations, which, in turn, is obtained by a search of the optimal approximation of the exact solution $U(\theta)$ [3]. For further details on constructing polynomial chaos and mapping eq. 13 to eq. 14, the reader is advised to refer to the original text book [5] where several examples are presented.

\subsection{Monte Carlo Simulation}

The third, most straightforward approach to SFEM is a merger of the Monte Carlo Simulation (MCS) with deterministic FEM. It is done with the following steps [2, 15]:

- adoption / recognition of sources of uncertainty,

- selection of random variables, analysis of their distributions and correlation,

- adequate generation of a set of random fields,

- construction of $N S$ deterministic FEM model variants in accordance with the generated fields ( $N S$ - number of model variants, number of simulations),

- calculation of model responses in $N S$ cases,

- $\quad$ statistical analysis of responses.

The last step is done with the use of formulas known from statistics. For example, the estimators of mean $\bar{u}_{i}$ and standard deviation $s_{u_{i}}$ of the analysed response (e.g. displacement $\left.u_{i}\right)$ in an $i$-th degree of freedom of FEM model are $[3,6]$ :

$$
\begin{gathered}
\bar{u}_{i}=\frac{1}{N S} \sum_{j=1}^{N S} u_{i}(j) \\
s_{u_{i}}=\sqrt{\frac{\sum_{j=1}^{N S}\left[\left(u_{i}(j)\right)^{2}-N S \cdot\left(\bar{u}_{i}\right)^{2}\right]}{N S-1}}
\end{gathered}
$$

The accuracy of these estimations increases with an increasing number of simulations $N S$. On the other hand, large $N S$ can lead to computational issues (especially for complex numerical models). For that reason, a number of sampling techniques, e.g. Latin Hyper Cube 
Sampling (LHS) and Improved LHS, were developed. Their use may lead to the reduction of $N S$ while maintaining an acceptable level of procedure's accuracy.

\subsection{Other probabilistic methods for analysis of FE model multiple responses}

Not only the Monte Carlo Simulation is based on the multiple analyses of the deterministic FE model. A generalized variation of SFEM introduced by Kamiński [11] in a form of the Response Function Method (RFM) is one of them. Using such an approach, several real-life engineering structures were successfully analysed, e.g. [16, 17].

In this context, two other important methods should be mentioned here: Response Surface Method (RSM) [18] and Point Estimate Method (PEM) [19, 20]. Both rely on the approximation of the model's response and are widely used in civil engineering problems. However, it should be empasiszed that these techniques are not classified as SFEM methods in [2] and [3].

\section{SFEM applications in bridge analysis}

The results of the author's extensive review of the works regarding SFEM applications in the assessment of bridges are collected in Table 1. As demonstrated, the cited works contain bridge analysis issues related to, e. g.: bridge-vehicle interaction and weight in motion problems, dynamic response of pedestrian bridges, probabilistic seismic analysis, reliability and fatigue reliability issues, influence of wind excitations, and local failure of bridge components.

Table 1. Application of probabilistic FE methods in analysis of bridge structures.

\begin{tabular}{|c|c|c|c|}
\hline method(s) used & object / element & aspect of analysis & ref. \\
\hline Perturbation SFEM & cable-stayed bridge & $\begin{array}{c}\text { prob. assessment of displacements and } \\
\text { internal forces (random mat. properties) }\end{array}$ & {$[21]$} \\
\hline Perturbation SFEM & $\begin{array}{c}\text { three-span, box } \\
\text { girder bridge }\end{array}$ & $\begin{array}{c}\text { stochastic seismic analysis (random material } \\
\text { properties) }\end{array}$ & {$[22]$} \\
\hline $\begin{array}{c}\text { Perturbation SFEM } \\
\text { (RFM) }\end{array}$ & $\begin{array}{c}\text { arch, pedestrian } \\
\text { bridge }\end{array}$ & $\begin{array}{c}\text { eigenfrequencies, displacements (uncertain } \\
\text { dynamic load / deck stiffness / material } \\
\text { properties) }\end{array}$ & {$[16]$} \\
\hline SSFEM & $\begin{array}{c}\text { single-span beam, } \\
\text { road bridge }\end{array}$ & $\begin{array}{c}\text { bridge-vehicle interaction (uncertain material } \\
\text { parameters) }\end{array}$ & {$[23]$} \\
\hline SSFEM & $\begin{array}{c}\text { single-span beam, } \\
\text { road bridge }\end{array}$ & $\begin{array}{c}\text { bridge-vehicle interaction (uncertain dynamic } \\
\text { loads, material density, elastic modulus, } \\
\text { damping) }\end{array}$ & {$[24]$} \\
\hline $\begin{array}{c}\text { (K-L) expansion } \\
\text { SFEM + MCS }\end{array}$ & $\begin{array}{c}\text { single-span, } \\
\text { laminated } \\
\text { composite beam }\end{array}$ & $\begin{array}{c}\text { bridge-vehicle interaction (randomness in } \\
\text { material properties and moving loads) }\end{array}$ & {$[25]$} \\
\hline K-L) expansion & $\begin{array}{c}\text { single-span beam, } \\
\text { road bridge }\end{array}$ & $\begin{array}{c}\text { bridge-vehicle interaction; moving force } \\
\text { identification (random excitation and material } \\
\text { properties) }\end{array}$ & {$[26]$} \\
\hline FFEM & $\begin{array}{c}\text { fable of suspension } \\
\text { bridge }\end{array}$ & $\begin{array}{c}\text { probability distribution of cable failure } \\
\text { (uncert. cable strength as 3D rand. field) }\end{array}$ & {$[27]$} \\
\hline FEM+MCS (LHS) & $\begin{array}{c}\text { steel, road bridge } \\
\text { (single-span, two } \\
\text { girders) }\end{array}$ & $\begin{array}{c}\text { fatigue reliability assessment (uncertain } \\
\text { vehicle loads in several aspects) }\end{array}$ & {$[28]$} \\
\hline
\end{tabular}




\begin{tabular}{|c|c|c|c|}
\hline FEM+MCS (LHS) & $\begin{array}{c}\text { three-span, } \\
\text { suspension bridge }\end{array}$ & $\begin{array}{c}\text { damping and flutter speed (structural and } \\
\text { aerodynamic uncertainties) }\end{array}$ & {$[29]$} \\
\hline FEM+MCS & $\begin{array}{c}\text { three-span, } \\
\text { suspension bridge } \\
\text { (as in [29]) }\end{array}$ & $\begin{array}{c}\text { seismic analysis (uncertainties in seismic } \\
\text { excitation) }\end{array}$ & {$[30]$} \\
\hline $\begin{array}{c}\text { FEM+MCS } \\
\text { RSM }\end{array}$ & $\begin{array}{c}\text { two PC box girders, } \\
\text { four-span, road } \\
\text { bridge }\end{array}$ & $\begin{array}{c}\text { FE model validation (uncertain material } \\
\text { properties, stiffness of support bearings and } \\
\text { expansion joints, stiffness between two } \\
\text { adjacent box-girders) }\end{array}$ & {$\left[\begin{array}{c}{[31]} \\
{[32]}\end{array}\right.$} \\
\hline $\begin{array}{c}\text { FEM+MCS } \\
\text { RSM }\end{array}$ & masonry arch bridge & $\begin{array}{c}\text { stochastic response, namely stresses and } \\
\text { displacements (random ground motion, } \\
\text { uncertain material properties) }\end{array}$ & {$[33]$} \\
\hline $\begin{array}{c}\text { FEM+MCS } \\
\text { RSM }\end{array}$ & $\begin{array}{c}\text { large-span } \\
\text { suspension bridge }\end{array}$ & $\begin{array}{c}\text { Probabilistic free vibration and flutter analysis } \\
\text { (uncertain material properties and stiffness) }\end{array}$ & {$[34]$} \\
\hline $\begin{array}{c}\text { FEM+ improved } \\
\text { RSM } \\
\text { (not SFEM) }\end{array}$ & $\begin{array}{c}\text { large-span } \\
\text { suspension bridge }\end{array}$ & $\begin{array}{c}\text { risk assessment for the construction phases } \\
\text { (random cross-section area of hangers and } \\
\text { cables, elasticity of cables) }\end{array}$ & {$[35]$} \\
\hline $\begin{array}{c}\text { FEM+RSM } \\
\text { (not SFEM) }\end{array}$ & arch bridge & $\begin{array}{c}\text { system reliability assessment (uncertain } \\
\text { section area, inertia, and resistant moments of } \\
\text { the girders) }\end{array}$ & {$[36]$} \\
\hline
\end{tabular}

\section{Computer implementation, feasibility issues}

Although the range of SFEM applications in the probabilistic assessment of bridge structures seems wide, some limitations of this approach were identified, especially in regard to the level of computer implementation. There are not many widely available or commercial computer programs that implement the full SFEM procedure in the variant of SSFEM or Perturbation SFEM. Examples of such are SFESTA and SFEDYN. These programs were developed by Kleiber and Hien [4] in the Fortran ${ }^{\circledR}$ programming language. Both tools cover the scope of a deterministic, stochastic and sensitivity analysis with the use of the perturbation approach. The first one concerns the static analysis of $3 \mathrm{D}$ trusses and the second the dynamic analysis of 3D frames. Only one-dimensional elements can be used (no plate, shell or block elements can be used). These tools were further used and developed for the probabilistic computations of bridges by the authors of [21] and [22]. The presented results of a probabilistic seismic analysis of a cantilever bridge and a cable-stayed bridge indicate the high efficiency of perturbation SFEM in relation to MCS. However, to the author's knowledge, the aforementioned computer program, even after developments, does not have capabilities of using finite elements other than one-dimensional ones. Therefore, higher-class models (e2s2, e2s3, or e3s3) or the ones that use several element types are not applicable.

The spectral version of SFEM (SSFEM) is also used for rather simple structural models [23-26]. Despite the author's efforts in the literature review, no higher-class models of bridges were found to be evaluated be means of SSFEM. However, it should be emphasized that there are some open-source MATLAB ${ }^{\circledR}$ implementations of SSFEM available e.g. [37, 38], which can be further developed and modified in the future.

Advanced models with different types of finite elements - which are most commonly used in real-life bridge engineering - are still analysed by these SFEM variants which are based on multiple calculations of a deterministic model (which is often prepared in commercial FEM systems e.g. MIDAS $\AA$, LUSAS $\AA$, DIANA $\AA$, ARSAP $\AA$, SOFiSTiK $\AA$, SCIA ${ }^{\circledR}$, ATENA ${ }^{\circledR}$, CivilFEM $\AA$, RM Bridge $\AA$, ANSYS $\AA$, ABAQUS $\AA$, etc.) with later analysis of adequate responses. It is in particular MCS and RFM. Also, RSM (which is implemented to e.g. ANSYS ${ }^{\circledR}$ ) and PEM are still very popular, and seem to be important alternatives to SFEM in many aspects of bridge infrastructure assessment computations. 


\section{Summary}

Probabilistic tools are more and more often used for an analysis of bridge objects. It is not only about their traditional use, for example for the design of standards, but also about the analysis of specific bridge infrastructure objects in order to adequately represent sources of uncertainty. An important part of these methods is SFEM, which is implemented in three main versions: perturbation SFEM, SSFEM and FEM + MCS. The scope of applications of these methods in assessing bridges is wide. It concerns, among others, reliability, dynamic (mass in motion, bridge-vehicle interaction), seismic, wind load or static structural (including local effects analysis) analyses. Unfortunately, the implementation and use of the full SFEM procedure in a perturbation or spectral variant usually requires the creation of an individual computer code. Therefore, many of the collected examples refer to very simple FEM models. In the case of more sophisticated FE models and the usage of deterministic, commercial FEM programs well-known to bridge engineers, MSC and RFM seem to be a valuable solution. In many aspects, a strong alternative to the last two mentioned variants of SFEM are still RSM and PEM.

This paper was partially financed by a subsidy for the maintaining of research potential and the financing of young scientists (University of Bielsko-Biała, Poland, Faculty of Materials, Civil and Environmental Engineering)

\section{References}

1. D. Gransberg, C. Lopez, D. Humphrey, J. of Constr. Eng. and Manag., 133(6), 404-408, Jun. (2007)

2. J. D. Arregui-Mena, L. Margetts, and P. M. Mummery, Arch. of Comput. Meth. in Eng., 23(1), 171-190, Mar. (2016)

3. G. Stefanou, Comp. Methods in Appl. Mech. and Eng., 198, 9-12 (2009)

4. M. Kleiber and T. D. Hien, The Stochastic Finite Element Method: Basic Perturbation Technique and Computer Implementation. Wiley (1993)

5. R. Ghanem and P. Spanos, Stochastic Finite Elements - a spectral approach., 2nd-nd ed. Dover Publications (2002)

6. A. Nowak and K. Collins, Reliability of Structures, International. McGraw-Hill Higher Education (2000)

7. W. E. Boyce and B. E. Goodwin, J. of the Soc. for Ind. and Appl. Math., 12(3), 613-629 (1964)

8. G. C. Hart and J. D. Collins, 'The treatment of randomness in finite element modelling', presented at the SAE shock and vibration symposium, Los Angeles, 2509-2519 (1970)

9. T. K. Hasselman and G. C. Hart, J. of the Eng. Mech. Div., 98, 561-579 (1972)

10. B. Huang, J. Liu, and H.-B. Gao, 'Statistic eigenvalue analysis of engineering structures based on substructure recursive stochastic finite element method', presented at the 9th International Symposium on Structural Engineering for Young Experts, Fuzhou \& Xiamen, China, 1, 18-21 (2006)

11. M. Kamiński, The Stochastic Perturbation Method for Computational Mechanics, 1st-st ed. Viley (2013)

12. K. Karhunen, Über lineare Methoden in der Wahrscheinlichkeitsrechnung. Sana (1947)

13. M. Loeve, Probability Theory I, 4th ed. New York: Springer-Verlag (1977)

14. M. Kac and A. J. F. Siegert, Ann. Math. Statist., 18(3), 438-442, Sep. (1947) 
15. A. Haldar and S. Mahadevan, Reliability Assessment Using Stochastic Finite Element Analysis, 1 edition. New York: Wiley (2000)

16. J. Szafran and M. Kamiński, Int. J. of Appl. Mech. and Eng., 22(1), (2017)

17. M. M. Kamiński and P. Świta, Comp. \& Str., 89(11-12), 1241-1252, Jun. (2011)

18. G. E. P. Box and K. B. Wilson, J. of the Royal Stat. Soc., 13(1), 1-45 (1951)

19. E. Rosenblueth, Point estimates for probability, 72 (1975)

20. C. Che-Hao, T. Yeou-Koung, and Y. Jinn-Chuang, Appl. Math. Model., 19(2), 95-105, Feb. (1995)

21. Ö. Çavdar, A. Bayraktar, and S. Adanur, Prob. Eng. Mech., 25(2), 279-289, Apr. (2010)

22. Ö. Çavdar, A. Bayraktar, and A. C. Altunışık, Civil Eng. and Envir. Sys., 32(3), 193205, Jul. (2015)

23. S. Q. Wu and S. S. Law, Prob. Eng. Mech., 26(2), 281-293, Apr. (2011)

24. S. Q. Wu and S. S. Law, Mech. Sys. and Sig. Proc., 27, 576-589, Feb. (2012)

25. T.-P. Chang, Appl. Math. and Comput., 242, 20-35, Sep. (2014)

26. S. Q. Wu and S. S. Law, Eng. Str., 32(4), 1016-1027, Apr. (2010)

27. A. Montoya, G. Deodatis, R. Betti, and H. Waisman, J. of Comp. in Civil Eng., 29, Aug. (2014)

28. D. M. Frangopol, A. Strauss, and S. Kim, J. of Bridge Eng., 13(3), 258-270, (2008)

29. T. Argentini, A. Pagani, D. Rocchi, and A. Zasso, J. of Wind Eng. and Ind. Aero., 128, 90-104, May (2014)

30. L. Sgambi, E. Garavaglia, N. Basso, and F. Bontempi, Eng. Str., 78, 100-111, Nov. (2014)

31. Z. Zong, X. Lin, and J. Niu, J. of Traf. and Transp. Eng. (Engl. Ed.), 2(4), 258-278, Aug. (2015)

32. X. Lin, Z. Zong, and J. Niu, J. of Traf. and Transp. Eng. (Engl. Ed.), 2(4), 279-289, Aug. (2015)

33. K. Hacıefendioğlu, H. B. Başağa, and S. Banerjee, Constr. and Buil. Mat., 134, 199209, Mar. (2017)

34. J. Cheng and R. Xiao, Eng. Str., 27(10), 1509-1518, Aug. (2005)

35. T. Cho and T. S. Kim, Finite El. in An. and Design, 44(6-7), 383-400, Apr. (2008)

36. A. S. Nowak and T. Cho, J. of Constr. Steel Res., 63(12), 1561-1569, Dec. (2007)

37. A. Der Kiureghian, T. Haukaas, and K. Fujimura, Struct. Saf., 28(1), 44-67, Jan. (2006)

38. F. U. Castillo, 'Probabilistic analysis of structures using stochastic finite elements', Masters Thesis, Universidad Nacional de Colombia (2015) 